\title{
Induction of Basal Resistance by Methyl Jasmonate against Fusarium culmorum in Bread Wheat
}

\author{
P. Motallebi ${ }^{1,2}$, S. Tonti ${ }^{2 *}$, V. Niknam ${ }^{1}$, H. Ebrahimzadeh ${ }^{1}$, A. Pisi ${ }^{2}$, P. Nipoti ${ }^{2}$, \\ M. HASHEMI ${ }^{3}$ and A. PrODI ${ }^{2}$ \\ ${ }^{1}$ Department of Plant Biology, Center of Excellence in Phylogeny of Living Organisms, School of Biology, \\ College of Science, University of Tehran, 14155-6455 Tehran, Iran \\ ${ }^{2}$ Department of Agricultural Sciences, Alma Mater Studiorum University of Bologna, \\ Viale Giuseppe Fanin 40, 40127 Bologna, Italy \\ ${ }^{3}$ Department of Plant Pathology, Seed and Plant Improvement Institute, Karaj, Iran \\ (Received 10 May 2016; Accepted 25 October 2016; \\ Communicated by J. Kolmer)
}

\begin{abstract}
Fusarium culmorum is a soilborne fungal pathogen, agent of crown and root rot disease (FCRR), responsible of major economic losses in wheat plants. This host-pathogen interaction, following methyl jasmonate (MeJA) application at the beginning of the necrotrophic stage of infection, has not been previously studied at molecular level. In this study, using real-time quantitative PCR, the emerging role of MeJA in the basal resistance of two bread wheat cultivars against $F$. culmorum has been investigated. MeJA treatment was dispensed 6 hours after pathogen inoculation (6 hai) to detect the defense response at the beginning of the necrotrophic stage. The expression of phenylalanine ammonia-lyase (PAL), lipoxygenase (LOX), cytochrome P450 (CYP709C1) genes and of some pathogenesis related (PR) genes, including PR3, PR4 and PR9, was examined in both root and crown tissues of the susceptible wheat cultivar Falat and the tolerant cultivar Sumai3. The pathogen responsive defense genes were induced in both cultivars, with a higher level of induction in Sumai3 than in Falat. MeJA treatment reduced the symptoms in cv Falat, whereas no significant effects have been detected in cv Sumai3. In fact, MeJA treatment caused a striking difference in defense gene induction. The genetic change was present in root and crown tissues of both wheat cultivars, demonstrating a systemic signaling pathway. The chemically induced protection correlated with induction of the F. culmorum-responsive genes supports a possible role of jasmonate signaling in regulating basal resistance in wheat $-F$. culmorum interaction.
\end{abstract}

Keywords: Fusarium culmorum, systemic acquired resistance, methyl jasmonate, realtime quantitative PCR, Triticum aestivum

\section{Introduction}

The ascomycete Fusarium culmorum (W.G. Smith) Saccardo is one of the hemibiotrophic pathogens with a short biotrophic phase preceding necrotrophism (Petti et al. 2012). It causes crown and root rot disease (FCRR) in many wheat growing countries (Caruso et

\footnotetext{
*Corresponding author; E-mail: stefano.tonti@crea.gov.it
} 
al. 1999; Backhouse and Burgess 2002; Beccari et al. 2011) including Iran (Motallebi et al. 2015a). The symptoms of FCRR often consisted of damping off, brown discoloration in root and crown tissues and whiteheads formation (Scherm et al. 2013). This pathogen is also one of the main causal agents of Fusarium head blight (FHB), another wheat disease, responsible of decreasing of seed quality due to mycotoxin presence. F. culmorum can produce nivalenol (NIV) and deoxynivalenol (DON) which are health threats for both humans and livestock (Hope and Magan 2003). Improving resistance of wheat cultivars to Fusarium diseases has become the major goal because of the serious threat to food safety. Based on the complex genetic nature of plants, the researchers have not been yet entirely successful to improve genetically disease resistance.. It has been shown that several genes, for example, wheat pathogenesis related (PR) genes by $F$. pseudograminearum (Desmond et al. 2006), expression of plant P450 gene "CYP709C1" by F. asiaticum (Li et al. 2010), lipoxygenase (LOX) and phenylalanine ammonia-lyase (PAL) genes during infection of wheat heads by F. graminearum, are induced in wheat plants ( $\mathrm{Li}$ and Yen 2008). Beccari et al. (2011) demonstrated that some host defense-associated genes, such as Wheatwin 1-2 (PR4), PR1, Chitinase (PR3), PAL, wheat induced resistance gene1 (WIR1) and LOX, against $F$. culmorum in wheat were induced in the early infection stages while decreased during the later stages. The plant itself induces production of phytohormones to control pathogen penetration and spread. Jasmonic acid (JA) and its derivatives such as methyl jasmonate (MeJA) are some of the key signal molecules devoted in regulating plant resistance to diverse pathogens and, as well as salicylic acid (SA), are associated with the necrotrophic and hemibiotrophic pathogens (Desmond et al. 2006; Makandar et al. 2012). JA-regulated mechanisms are primarily involved in defense from necrotrophic pathogens, while SA is mainly engaged with pathogens that display a biotrophic phase (Glazebrook 2005).

Ding et al. (2011) demonstrated that the resistance to hemibiotrophic F. graminearum infection is associated to coordinated and ordered expressions of diverse defense signaling pathways and to altered secondary metabolism. They proposed a model to illustrate the early cellular events leading to FHB resistance, with two major phases of resistance reactions. The first phase occurred within 6 hours after inoculation (6 hai), probably corresponding to a transient biotrophic stage, and it was characterized by changes or activation of $\mathrm{Ca}^{2+}$ fluxes, SA signaling, Reactive Oxygen Species (ROS) production and scavenging. The second phase occurred after 6 hai, probably corresponding to the beginning of the necrotrophic stage, and it was marked by the activation of JA signaling pathway. F. graminearum growth induces multiple mechanisms that are activated during the necrotrophic stage. The resistance related activities, such as PR defense genes, cell wall enforcement with lignin biosynthesis, antimicrobial compound synthesis and deoxynivalenol detoxification by UDP-glycosyltransferase, could start after fungal infection. The activating order of these signaling events in inducing resistance, limiting the programmed cell death (PCD), could be critical. JA and SA are involved in the systemic activation of defenses. SA signalling, compared with JA, acts earlier during the infection limiting $F$. graminearum infection, thus supporting the suggestion that both are required for the full level of basal resistance against F. graminearum (Makandar et al. 2012). The basal 
resistance of the plant is the defense that reduces pathogen infection after successful penetration by inducing basic defense response genes during early infection, or by accelerating the cellular death of cells close to the infection site preventing the advance of infection and/or by generating a toxic environment that inhibits the growth of the pathogen inside the cells. In spite of the importance of FCRR disease in agriculture, only few studies have dealt with it and the basis for resistance against FCRR is still unknown. In our previous research, we have demonstrated the effects of MeJA on wheat defense gene expression during the later stages of $F c$-infection in wheat (Motallebi et al. 2015b). The data suggested that MeJA is a potent inducer of defense gene expression particularly 2 days post inoculation (2 dpi) which was coincided with necrotrophic stage. However, based on the hemibiotrophic lifestyle, 6 hai, which is a switch time between SA/JA pathways, seems to be critical for the regulation of the basal resistance (Ding et al. 2011). Hence, in this current study for the first time, we have used real-time quantitative PCR (RT-qPCR) to measure expression of a panel of wheat defense genes following MeJA treatment at significant earlier time point probably corresponding to the start of the necrotrophic stage of $F c$-infection. This has been applied to study defense genes expression by MeJA treatment at 6 hai.

Indeed, the previous work (Motallebi et al. 2015b) has been extended to this study, showing that this differential response exists in wheat genotypes between the beginning of the necrotrophic and during necrotrophic stage of F. culmorum infection. Real-time quantitative PCR has been used to compare the systemic expression of wheat defenseassociated genes such as PAL, LOX, cytochrome P450 gene (CYP709C1), PR3, PR4, and peroxidase (PR9) in two bread wheat cultivars, Falat and Sumai3 with different resistance levels to FCRR. The effect of exogenous application of MeJA, as a key factor in systemic acquired resistance (SAR), has been investigated at 6 hai (probably the beginning of the necrotrophic stage) to enable the improvement of basal resistance on wheat cultivars through the quantification of the F. culmorum responsive genes expression and FCRR disease development.

\section{Materials and Methods}

\section{Wheat plants and F. culmorum strain F3}

Seeds of Iranian spring bread wheat (Triticum aestivum L.) cultivar Falat and Chinese cultivar Sumai3 were used for the two experimental trials. The first to evaluate FCRR development, the second to examine the expression of wheat defense-associated genes after MeJA application and F. culmorum inoculation. Eight seeds were sown in each pot filled with sterile soil, at approximately $2 \mathrm{~cm}$ below the soil surface, to carry out the experimental trials.

F. culmorum strain F3, isolated from roots of wheat cultivated in Iran and molecularly identified (Motallebi et al. 2015a), was used. The inoculum was produced as described in Motallebi et al. (2015a). 


\section{F. culmorum inoculation and MeJA treatment of wheat seedlings}

The suspension of F. culmorum macroconidia, along with the mycelium and V8 medium, was filtered through a sterile syringe filled with double layers of autoclaved cheesecloth, for both trials. Macroconidia concentration was determined using a haemocytometer and the suspension adjusted to $2 \times 10^{6}$ macroconidia/ml. The plants, 2-week-old, were inoculated by removing the soil around the collar and applying $100 \mu \mathrm{l}$ of $F$. culmorum suspension, or water for control plants, both containing $0.02 \%(\mathrm{v} / \mathrm{v})$ Tween 20.

The defenses were chemically activated by irrigating wheat plants with MeJA. MeJA (Sigma-Aldrich) stock solution $(1 \mathrm{mM})$ was prepared in $0.1 \%$ ethanol and $200 \mu \mathrm{M}$ of MeJA were poured with a pipette directly into the soil around each wheat seedling 6 hours after F. culmorum inoculation. Control plants were treated at the same time with $0.1 \%$ water ethanol solution.

The soil was watered every two days and the plants were grown in a growth chamber, at $22{ }^{\circ} \mathrm{C} / 18{ }^{\circ} \mathrm{C}$ day/night with $60-70 \%$ relative humidity and $16 \mathrm{~h}$ photoperiod (light intensity 10,000 lux). In addition, the effect of MeJA on the growth of F. culmorum was also assayed at the beginning of this project. The test was performed by adding MeJA (200 $\mu \mathrm{M})$ to PDA Petri dishes. The results indicated that MeJA did not significantly affect the growth of F. culmorum in vitro (data not shown).

\section{Experimental trial}

For disease symptom evaluation (first trial) 144 pots, with 8 plants each, were prepared and divided into 2 treatments: one inoculated with $F$. culmorum $(F c)$ and the other with $F_{C}+$ MeJA. Each treatment consisted on four pots for each 3 evaluation-times - 7, 14 and 21 days post inoculation (dpi) - in a complete randomized design with three repetitions for each cultivar ( 4 pots $\times 3$ evaluation-times $\times 3$ repetitions $\times 2$ wheat cultivars).

For real-time qPCR assay (second trial) 144 pots, with 8 plants each, were prepared and divided into 4 treatments: Ctr (control plants); MeJA (plants treated with MeJA); $F_{c}$ (plants inoculated with $F$. culmorum) and $F_{C}+$ MeJA (plants inoculated with $F_{c}$ and treated with MeJA). Each treatment consisted on three pots for each 2 evaluation times -24 and 48 hai - in a complete randomized design with three repetitions for each cultivar ( 3 pots $\times 2$ evaluation times $\times 3$ repetitions $\times 2$ wheat cultivars).

\section{Disease symptom evaluation}

For each evaluation time, the plants were gently uprooted and crown disease symptoms were assessed based upon a five-point scale (class $0=$ healthy stem; class $1=$ mild browning on the stem; class $2=$ browning on one-half of the stem; class $3=$ complete browning of the stem; class $4=$ (dead plant).

The disease severity $(D S)$ for each treatment was calculated using McKinney formula (1923):

$$
D S=\left[\sum\left(c^{*} f\right) / n^{*} N\right]^{*} 100
$$


where $c=$ disease class, $f=$ frequency, $n=$ number of observed plants and $N=$ the highest value of the empirical scale.

\section{Real-time quantitative PCR assay}

Crown and root tissues of all the plants were harvested for real-time qPCR assay. Approximately $3-\mathrm{cm}$ of each root around the inoculated region and 2-cm of the basal stem (crown tissue) were used to determine genes expression in roots and crowns. Inoculated samples were collected at 1 and 2 (dpi) (24 and 48 hpi), while those without $F c$-inoculation, at 18 and 42 hours post chemical treatment (hpt).

The harvested root and crown tissues were immediately frozen in liquid nitrogen and stored at $-80^{\circ} \mathrm{C}$ until processing. For RNA extraction each sample was ground in sterile mortars. Plant RNA was extracted using the Sigma Plant Total RNA Kit (Sigma-Aldrich) according to Spectrum ${ }^{\mathrm{TM}}$ plant manufacturer's protocol. The DNase treatment was performed using the DNA-free kit (Sigma-Aldrich) according to the manufacturer's directions. Twenty-five ng of resulting RNA was used to synthesize cDNA using SuperScript III reverse transcriptase (Invitrogen) with $10 \mathrm{ng} / \mu \mathrm{l}$ Oligo (dT) primer. cDNAs were diluted 1:10 in RNA free water. RT-qPCR was performed in 96 well plates using an ABI7000 v 1.2.3 Sequence Detection System. Each reaction was carried out in a volume of $25 \mu 1$ containing $12.5 \mu \mathrm{l}$ GoTaq ${ }^{\circledR}$ qPCR Master Mix (Promega), $0.25 \mu \mathrm{l} \mathrm{CXR} \mathrm{(100X)} \mathrm{reference}$ dye, $1.5 \mu \mathrm{l}$ from each forward and reverse gene-specific primers (Table S1*), $4.25 \mu \mathrm{l}$ Nuclease-Free Water and $5 \mu 1$ of cDNA derived from the above-mentioned reverse transcriptions. PCRs amplification was performed under the following conditions: $95{ }^{\circ} \mathrm{C}$ for $10 \mathrm{~min}$, followed by 40 cycles at $95^{\circ} \mathrm{C}$ for $15 \mathrm{~s}$ and $62^{\circ} \mathrm{C}$ for $1 \mathrm{~min}$. Melting curve analysis was performed at the end of each reaction to confirm the amplification of genespecific products. Data were analysed using SDS1.2 software (Applied Biosystems). Exponential amplification was plotted on a logarithmic scale and Rn was set to 0.32 for each RT-qPCR plate to obtain the cycle threshold $(\mathrm{Ct})$ values. Control samples were always run on the same plate beside the treated samples. A wheat $\beta$-tubulin housekeeping gene (Table S1) was co-amplified as an internal control to normalize the total amounts of cDNAs present in each reaction and to eliminate the differences among the samples. Each sample was replicated three times, and the analysis of the target gene expression was determined using the $2^{-\Delta \Delta C T}$ method (Livak and Schmittgen 2001). All gene expression levels were expressed and compared to the appropriate non-inoculated controls.

\section{Statistical analysis}

Significant differences between $F_{C}$-inoculated plants and chemically treated plants $(F c+M e J A)$ were determined using a $t$-test. Normally distributed data were analysed using a one-way analysis of variance (ANOVA) and statistically significant differences $(P \leq 0.05)$ between samples were evaluated.

*Further details about the Electronic Supplementary Material (ESM) can be found at the end of the article. 


\section{Results}

\section{Disease development}

$F_{C}$-inoculated plants $\left(F_{C}\right)$ of $\mathrm{cv}$ Sumai3 were more resistant than those of cv Falat (Fig. S1), showing significant and lower Disease Severity $(D S)$ values than Falat plants. Plants of cv Falat with MeJA application at 6 hai $\left(F_{C}+\right.$ MeJA) showed significant lower DS values at 7 and 14 dpi than those $F c$. This difference was not statistically significant at 21 dpi (Fig. S1).

It is relevant to mention that statistical differences on DS values were not observed in cv Sumai 3 between $F c$ and $F c+$ MeJA plants at any evaluation time.

\section{RT-qPCR analysis of wheat defense genes in response to infection by $\mathrm{F}$. culmorum}

Comparing the two genotypes, all gene expressions were consistently greater, about ten times higher, in the tolerant genotype Sumai3 than in the susceptible one (Fig. S2).

In each cultivar, the gene expression, in wheat root tissue in direct contact with the pathogen, had the same pattern of that observed in the crown tissue, not in direct contact with the fungal hyphae except a striking difference in defense gene induction of CYP709C1 between root and crown of cv Falat (Fig. S2 A and B). In fact, this gene transcript was weakly induced in roots at $1 \mathrm{dpi}$, whereas it was not present in crown tissues at the same time.

Comparing the two sampling times it appeared that in cv Falat, five of six genes were induced to a higher level at 2 dpi with the exception of PAL which was higher at 1 dpi (Fig. S2 A and B). Likewise, in cv Sumai3 four of six genes were induced at higher level at 2 dpi with the exception of PR3 and PR9 which were higher at 1dpi (Fig. S2 C and D).

\section{RT-qPCR analysis of wheat defense genes in response to MeJA treatment}

All the six genes tested were induced after MeJA treatment in both cultivars (Fig. S3 A-D) and in each cultivar, the gene transcripts were present at similar levels in the crown and root tissues. At both evaluation times (18 and 42 hours post treatment), the values were not different in cv Falat except for PR9 where it was higher at 18 hpt (Fig. S3 A and B), while in cv Sumai3 the trend was higher at $42 \mathrm{hpt}$ except for PR9 where it was higher at $18 \mathrm{hpt}$.

Differential expression of defense genes by MeJA treatment after F. culmorum inoculation

All gene transcripts with MeJA treatment at 6 hai were present at similar levels in both crown and root tissues within the same cultivar (Fig. S4). This treatment caused a striking difference in defense gene expression between both genotypes: MeJA strongly induced expression of PR3, LOX and PAL transcripts approximately ten times higher in cv Falat compared to cv Sumai3 (Fig. S4), but repressed PR4, PR9 and CYP709C1 genes expres- 
sion in cv Falat (Fig. S4 A and B). The expression of LOX in cv Falat (Fig. S4 A and B) was 66.56- and 39.55-fold higher than in the control plants at 1 and 2 dpi (Fig. S3 A and B), respectively, while in cv Sumai3 (Fig. S4 C and D) was about 24- and 9-fold, respectively. Indeed, MeJA applied at 6 hai improved JA signaling pathway in wheat, for most of the pathogen inducible genes particularly in cv Falat, signifying earlier induction with a higher level at 1 dpi (Fig. S4 A and B). In cv Sumai3, MeJA induced a weak transcription of all selected genes and the highest levels of induction for most pathogen inducible genes were detected at 2 dpi, with the exception of LOX gene (Fig. S4 C and D). The induction for PR4, which is one of the remarkable genes in defense response, was about 10 times higher in cv Sumai3 than in cv Falat, while this induction level in both cultivars was approximately 10 -fold lower after MeJA treatment compared to F. culmorum inoculated ones (Fig S2).

\section{Discussion}

Resistance against F. culmorum, the causal agent of FCRR, has attracted a great deal of attention. Even if there are several studies on wheat-pathogen interaction (Desmond et al. 2006; Makandar et al. 2010; Beccari et al. 2011; Makandar et al. 2012; Motallebi et al. 2015b), little is known about the contribution of JA derivatives, such as MeJA, to the resistance mechanisms. In the current work, several genes expression has been investigated to better understand the differences between susceptible and tolerant wheat genotypes infected by F. culmorum. This study reports for the first time the effect of MeJA application in basal resistance, the defense that reduces pathogen infection after successful penetration and onset of disease, of wheat against F. culmorum and it has been shown, based on the hemibiotrophic pathogen lifecycle, that at 6 hai there is a switch between SA/JA pathways, probably corresponding to the beginning of the necrotrophic stage of infection. This could be a critical time-point for the regulation of the basal resistance to FCRR in wheat. It is well known that JA and SA are involved in the systemic activation of defenses in dicotyledons and monocotyledons (Makandar et al. 2012). Generally JA is a signal molecule required for the activation of induced systemic resistance (ISR) while SA signal pathway is required for systemic acquired resistance (SAR) activation. JA is mainly involved in inducing resistance against necrotrophic pathogens (Verhagen et al. 2004), while SA towards the biotrophic ones (Durrant and Dong 2004; Chaturvedi and Shah 2007). Despite the well-documented antagonism between the two signaling molecules, both SA and JA are required for a full resistance against necrotrophic and hemibiotrophic pathogens (Desmond et al. 2006; Ding et al. 2011; Makandar et al. 2012).

Makandar et al. (2010) proposed a primary role of SA signal pathway in wheat resistance towards $F$. graminearum during the early stage of hemibiotrophic interaction. The same authors, following the impact of MeJA treatment on FHB severity in wheat, showed a reduction in FHB symptoms in cv Bobwite spikes, when exposed to MeJA vapour after the inoculation. This behaviour was not shown when the plants were exposed prior or during the early stage of fungal infection. Ding et al. (2011) measured the endogenous level of SA and MeJA in spikes FHB infected with F. graminearum, and they demonstrated an 
increase of SA within $3 \mathrm{hpi}$ and of MeJA, with a peak at $12 \mathrm{hpi}$, in the resistant cv Wangshuibai compared to a sensible mutant. All these observations were explained supposing a model for monocotyledons where the induction of SA signal was temporally followed by MeJA mediated pathway increase, leading to a resistant reaction; however MeJA exogenous application decreased FHB severity only in a susceptible genotype and did not change the behaviour of the resistant cultivar (Makandar et al. 2012). Similarly, our results strongly indicate that MeJA, when applied as a soil drench at 6 hai, effectively decreased disease severity only in the susceptible wheat cv Falat in the first two weeks and did not affect Sumai3, the tolerant cultivar one (Fig. S1). Our previous research has reported that exposure of wheat genotypes to MeJA during the later stages of infection beginning at $1 \mathrm{dpi}$ and later promoted resistance, while when applied prior to inoculation or at the time of fungal inoculation, attenuated FCRR resistance (Motallebi et al. 2015b). Whereas, present results using MeJA treatment at 6 hai clearly indicate that the induction of defense genes in wheat occurred at the beginning of the necrotrophic stage in a genotype dependent manner. Studies of genes expression in wheat provide genetic evidence confirming a critical role for MeJA in basal resistance to FCRR. The aim of this study was to get more information on the mechanisms involved in the complex nature of overall wheat resistance to root and crown rot pathogens, particularly during the early stage of infection.

Hence, one of our main goal was to induce resistance against $F c$-infection and therefore to test the ability of MeJA to promote the defense genes expression. The inductions of six defense response genes (PR3, PR4, PR9, LOX, PAL and CYP709C1) after F. culmorum inoculation were detected in both cultivars. However, the expressions of these genes had higher level in cv Sumai3 than in Falat. This confirmed that highly regulated host defense response in wheat seedlings was triggered by the hemibiotrophic F. culmorum pathogen. An opposite behavior between the cultivars was observed after MeJA treatment, cv Falat showed a gene transcription higher than cv Sumai3. MeJA induced all the six defense response genes tested in both cultivars (Fig. S3), but at 6 hai, the three defense response genes, PR4, PR9 and CYP709C1, were not induced in cv Falat (Fig. S4 A and B).

The expression of peroxidase is crucial to delay the cell damage (preferential way to obtain nutrients for necrotrophic fungi). Caruso et al. (1999) reported an increase in peroxidase activity associated to an early defense response during pathogen infection. PR4 gene encodes proteins showing antifungal activity towards pathogens (Caruso et al. 1999). In this work it was found that PR4 gene was induced by F. culmorum without chemical treatment, this in accord with Desmond et al. (2008) that reported PR4 induced at the base of the stem in wheat cultivars by F. pseudograminearum. Furthermore CYP709C1 encodes for cytochrome p450 related to a resistant response against fungal pathogens in wheat, and this transcript was observed in close association with resistance reaction in previous study on wheat challenged with $F$. asiaticum either in the spikes or seedlings ( $\mathrm{Li}$ et al. 2010). In fact, the ability of JA to attenuate activation of SA signaling (Pieterse et al. 2009) may explain the attenuation of PR4, PR9 and CYP709C1 conferring FCRR resistance in wheat. It is also important to take into account that some of the changes observed might be specifically induced by pathogens as part of their infection 
strategy (Bell and Mullet 1993). For instance, the effect of some fungal effectors may affect the trigged immunity system and repress the MeJA influence as a chemical inducer in the susceptible cultivars.

It has also been shown that disease severity was reduced over a 2-week period in cv Falat only, but the symptoms attenuation was not persistent, and no significant differences were seen between treated and untreated plants after 3 weeks, when a greater rate of symptom development was observed. The symptom delays in cv. Falat, suggest that the activation of JA signaling pathway may provide an increase resistance in wheat. Exogenous application of MeJA at the hypothetical shifting time between SA and JA pathway, promotes a basal resistance response in the susceptible cultivar confirming that SAR is important for FCRR resistance. It appears that the symptom delay in $\mathrm{cv}$ Falat is related to the up-expression of transcripts related to PR3, LOX and PAL genes, and unexpectedly repressed PR4 (Wheatwin1-2), PR9 (peroxidase) and cytochrome P450 gene (CYP709C1). Exogenous application of MeJA seems instead to suppress Sumai3 response, where the transcript abundance was almost 10 times less than that observed in the $F_{C}$ inoculated plants, even if it does not correspond to the visual symptom expression. In particular a decrease in peroxidase transcription and an increase of PAL should be related to an indirect decrease of ROS activity and to a direct enhancement of phenylpropanoid pathway.

PAL is a key plant enzyme at the entry point of the phenylpropanoid pathway. It should be noted that an increase in PAL activity may be related to the plant defense system through biosynthesis of active metabolites, such as phytoalexins, phenols, lignins, and SA in plant defense pathways (Mandal et al. 2009). Similarly, Chen et al. (2000) reported that high levels of PAL were induced in cucumber roots inoculated with Pythium aphanidermatum. LOX enzyme is responsible for membrane degradation by deoxygenation of polyunsaturated fatty acids. Both F. culmorum infection and MeJA treatment produced an increase in LOX expression in both cultivars. LOX enhancement could be related to biosynthesis of jasmonic acid (JA), which is involved in plant defense responses to necrotrophic pathogens (Creelman and Mullet 1997; Bari and Jones 2009). The current results suggest a positive feedback in JA production. Similarly, previous studies have indicated increased activity and expression of LOX against pathogen attack. Devi et al. (2000) and Mhaske et al. (2013) reported that LOX activity increased in castor (Ricinus communis) and pigeon pea (Cajanus cajan) resistant genotypes after pathogen inoculation. The increased LOX activity may generate signal molecules such as JA, methyl JA/or lipid peroxides, which coordinately trigger specific defense responses. The observation that PAL and LOX expression were strongly affected by the application of MeJA in both cultivars supported the idea that their basal resistance could be JA dependent. A similar phenomenon was observed by Desmond et al. (2006) using two wheat cultivars with different susceptibility to FCRR, where the most susceptible cultivar had a weak reaction to F. pseudograminearum, but a strong response to MeJA treatments.

Additionally, we found that accumulation of defense response gene transcripts is systemic in wheat upon F. culmorum infection in both susceptible and tolerant genotypes, because all the genes were also expressed in crown tissue which had not been yet in direct 
contact with either the fungus or MeJA, signifying that a form of systemic response was taking place in this interaction. These data indicate that the systemic induction of defense response genes is more likely a general host response to infection. Indeed, uninoculated crown tissue of inoculated roots expressed the same level of defense response genes.

In conclusion, this work has demonstrated that MeJA signaling contributes to basal resistance against FCRR in wheat. This study provides an overview and novel insights into the F. culmorum-wheat interaction. Many aspects of the response induced in plants, after pathogen infection, appear to have the potential to stop the fungus growth, even if the disease still occurs. Furthermore, is should not be neglected the host genetic background. MeJA treatment makes wheat cultivars more resistant to F. culmorum infection by altering the levels of the mentioned parameters. It is indicated that a central role (whether direct or indirect) for the MeJA signaling pathway in activating wheat defense response against FCRR is possible. The resistance-dependent expression patterns imply that FCRR resistance may share common signal transduction pathways for the activation of the defense genes in both crown and root tissues. Taking all these data into consideration this study strengthens the interpretation that MeJA- and pathogen-induced genes are regulated through the genotype dependent pathways in wheat. These results may serve as a foundation to understand mechanisms and regulations underlying FCRR resistant reactions in wheat and to reveal the functions of the MeJA, as a chemical treatment at the beginning of the necrotrophic stage of infection.

\section{Acknowledgements}

Our high gratitude goes to Dr. Makandar of the Department of Plant Sciences, University of Hyderabad, India, for her constructive advice and to Mr. Stefano Borsari for his technical assistance.

\section{References}

Backhouse, D., Burgess, L.W. 2002. Climatic analysis of the distribution of Fusarium graminearum, F. pseudograminearum and F. culmorum on cereals in Australia. Austr. Plant Pathol. 31:321-332.

Bari, R., Jones, J.D.G. 2009. Role of plant hormones in plant defense responses. Plant Mol. Biol. 69:473-488.

Beccari, G., Covarelli, L., Nicholson, P. 2011. Infection processes and soft wheat response to root rot and crown rot caused by Fusarium culmorum. Plant Pathol. 60:671-684.

Bell, E., Mullet, J.E. 1993. Characterization of an Arabidopsis lipoxygenase gene responsive to methyl jasmonate and wounding. Plant Physiol. 103:1133-1137.

Caruso, C., Chilosi, G., Caporale, C., Leonardi, L., Bertini, L., Magro, P., Buonocore, V. 1999. Induction of pathogenesis-related proteins in germinating wheat seeds infected with Fusarium culmorum. Plant Sci. 140:87-97.

Chaturvedi, R., Shah, J. 2007. Salicylic acid in plant disease resistance. In: Hayat, S., Ahmad, A. (eds), Salicylic Acid-A Plant Hormone. Springer. Dordrecht, The Netherlands. pp. 335-370.

Chen, C., Belanger, R.R., Benhamou, N., Paulitz, T.C. 2000. Defense enzymes induced in cucumber roots by treatment with plant growth-promoting rhizobacteria (PGPR) and Pythium aphanidermatum. Physiol. and Mol. Plant Pathol. 56:13-23.

Coram, T.E., Wang, M.N., Chen, X.M. 2008. Transcriptome analysis of the wheat-Puccinia strifformis f.sp. tritici interaction. Mol. Plant Pathol. 9:157-169. 
Creelman, R.A., Mullet, J.E. 1997. Oligosaccharins, brassinolides and jasmonates: nontraditional regulators of plant growth, development, and gene expression. Plant Cell 9:1211-1223.

Desmond, O.J., Edgar, C.I., Manners, J.M., Maclean, D.J., Schenk, P.M., Kazan, K. 2006. Methyl jasmonate induced gene expression in wheat delays symptom development by the crown rot pathogen Fusarium pseudograminearum. Physiol. and Mol. Plant Pathol. 67:171-179.

Desmond, O.J., Manners, J.M., Schenk, P.M., Maclean, D.J., Kazan, K. 2008. Gene expression analysis of the wheat response to infection by Fusarium pseudograminearum. Physiol. and Mol. Plant Pathol. 3:40-47.

Devi, P.U.M., Reddy, P.S., Rani, N.U., Reddy, K., Reddy, M.N., Reddanna, P. 2000. Lipoxygenase metabolites of $\alpha$-linolenic acid in the development of resistance in Pigeon pea, Cajanus cajan (L.) Millsp, seedlings against Fusarium udum infection. Eur. J. Plant Pathol. 106:857-865.

Ding, L., Xu, H., Yi, H., Yang, L., Kong, Z., Zhang, L., Xue, S., Jia, H., Ma, Z. 2011. Resistance to hemibiotrophic $F$. graminearum infection is associated with coordinated and ordered expression of diverse defense signaling pathways. PLOS One, 6:e19008.

Durrant, W.E., Dong, X. 2004. Systemic acquired resistance. Annu. Rev. Phytopathol. 42:185-209.

Glazebrook, J. 2005. Contrasting mechanism of defense against biotrophic and necrotrophic pathogens. Annu. Rev. Phytopathol. 43:205-227.

Hope, R., Magan, N. 2003. Two-dimensional environmental profiles of growth, deoxynivalenol and nivalenol production by Fusarium culmorum on a wheat-based substrate. Letters in Appl. Microbiol. 37:70-74.

Li, G., Yen, Y. 2008. Jasmonate and ethylene signaling pathway may mediate Fusarium head blight resistance in wheat. Crop Sci. 48:1888-1896.

Li, X., Zhang, J.B., Song, B., Li, H.P., Xu, H.Q., Qu, B., Dang, F.J., Liao, Y.C. 2010. Resistance to Fusarium head blight and seedling blight in wheat is associated with activation of a cytochrome P450 gene. Phytopathol. 100:183-191.

Livak, K.J., Schmittgen, T.D. 2001. Analysis of relative gene expression data using real-time quantitative PCR and the $2^{-\triangle \Delta C T}$ method. Methods 25:402-408.

Makandar, R., Nalam, V., Chaturvedi, R., Jeannotte, R., Sparks, A.A., Shah, J. 2010. Involvement of salicylate and jasmonate signaling pathways in Arabidopsis interaction with Fusarium graminearum. Mol. PlantMicrobe Interactions 23:861-870.

Makandar, R., Nalam, V.J., Lee, H., Trick, H.N., Dong, Y., Shah, J. 2012. Salicylic acid regulates basal resistance to Fusarium head blight in wheat. Mol. Plant-Microbe Interactions 25:431-439.

Mandal, S., Mallick, N., Mitra, A. 2009. Salicylic acid-induced resistance to Fusarium oxysporum f.sp. lycopersici in tomato. Plant Physiol. and Biochem. 47:642-649.

McKinney, H.H. 1923. Influence of soil temperature and moisture on infection of wheat seedlings by Helminthosporium sativum. J. of Agric. Res. 26:195-217.

Mhaske, S.D., Mahatma, M.K., Jha, S., Singh, P., Ahmad, T. 2013. Polyamine metabolism and lipoxygenase activity during Fusarium oxysporum f. sp. ricini-castor interaction. Physiol. and Mol. Biol. of Plants 19:323-331.

Motallebi, P., Alkadri, D., Pisi, A., Nipoti, P., Tonti, S., Niknam, V., Hashemi, M., Prodi, A. 2015a. Biomolecular study on Iranian Fusarium culmorum strains on durum wheat and comparison with Italian and Syrian populations. Phytopathologia Mediterranea, 54:437-445.

Motallebi, P., Niknam, V., Ebrahimzadeh, H., Hashemi, M., Pisi, A., Prodi, A., Tonti, S., Nipoti, P. 2015b. Methyl jasmonate strengthens wheat plants against root and crown rot pathogen Fusarium culmorum infection. J. of Plant Growth Regulation 34:624-636.

Petti, C., Reiber, K., Ali, S.S., Berney, M., Doohan, F.M. 2012. Auxin as a player in the biocontrol of Fusarium head blight disease of barley and its potential as a disease control agent. BMC Plant Biol. 12:224.

Pieterse, C.M.J., Leon-Reyes, A., Van der Ent, S., Van Wees, S.C.M. 2009. Networking by small-molecule hormones in plant immunity. Nature Chem. Biol. 5:308-316.

Scherm, B., Balmas, V., Spanu, F., Pani, G., Delogu, G., Pasquali, M., Migheli, Q. 2013. Fusarium culmorum: causal agent of foot and root rot and head blight on wheat. Mol. Plant Pathol. 14:323-341.

Verhagen, B.W.M., Glazebrook, J., Zhu, T., Chang, H.S., Van Loon, L.C., Pieterse, C.M.J. 2004. The transcriptome of rhizobacteria induced systemic resistance in Arabidopsis. Mol. Plant-Microbe Interactions 17:895908 . 


\section{Electronic Supplementary Material (ESM)}

Electronic Supplementary Material (ESM) associated with this article can be found at the website of CRC at http://www.akademiai.com/content/120427/

Electronic Supplementary Table S1. Primer sequences used for gene expression study by RT-qPCR in wheat seedlings

Electronic Supplementary Figure S1. Disease severity (\%) in wheat cultivars (Falat and Sumai3) inoculated with Fusarium culmorum $(F c)$ and treated with MeJA at 6 hai $F c(F c+\mathrm{MeJA})$ at three evaluation times $(7,14$, $21 \mathrm{dpi})$. Error bars represent the standard error (SE) of means for three repetitions

Electronic Supplementary Figure S2. RT-qPCR analyses of defense-related gene transcripts (PR3, PR4, PR9, LOX, PAL, CYP709C1) in the roots (A and C) and crown (B and D) tissues of two weeks old wheat plants cv Falat (A and B) and Sumai3 (C and D) at 1 and 2 days post inoculation (dpi) with Fusarium culmorum. Columns represent average induction $( \pm \mathrm{SE})$ of gene transcripts, plotted on a logarithmic scale. Values have been normalized to wheat $\beta$-tubulin normalization factor

Electronic Supplementary Figure S3. RT-qPCR analyses of defense-related gene transcripts (PR3, PR4, PR9, LOX, PAL, CYP709C1) in the roots (A and C) and crown (B and D) tissues of two weeks old wheat plants cv Falat (A and B) and Sumai3 (C and D) at 18 and 42 hours post treatment with MeJA (hpt). Columns represent average induction $( \pm \mathrm{SE})$ of gene transcripts, plotted on a logarithmic scale. Values have been normalized to wheat $\beta$-tubulin normalization factor

Electronic Supplementary Figure S4. RT-qPCR analyses of defense-related gene transcripts (PR3, PR4, PR9, LOX, PAL, CYP709C1) in the roots (A and C) and crown (B and D) tissues of two weeks old wheat plants cv Falat (A and B) and Sumai3 (C and D) at 1 and 2 days post $F c$ inoculation (dpi) following MeJA treatment at 6 hours after inoculation (6 hai). Columns represent average induction $( \pm \mathrm{SE})$ of gene transcripts, plotted on a logarithmic scale. Values have been normalized to wheat $\beta$-tubulin normalization factor 